\title{
Joint BLAST-STTC for MIMO-OFDM System
}

\author{
Christine Niyizamwiyitira, Chul-Gyu Kang and Chang-Heon Oh. Author, Member, KIMICS
}

\begin{abstract}
This study focuses on improving MIMOOFDM systems by combining a wireless communication architecture known as vertical BLAST(bell laboratories layered space-time) or V-BLAST and STTC(space time trellis coding). In this paper, the combination is done by introducing STTC in each V-BLAST layer. Moreover, this architecture uses multiple antennas that are grouped into small number of antennas which makes it less complex to decode by decoding every group. Whereas, in traditional VBLAST, all the antennas form one group and they are decoded together at the receiver, therefore, this increases the complexity as the number of antennas is getting high.

We compare the bit error rate performance of this system with MIMO-OFDM that uses convolutional coding instead of STTC. Under the same spectral efficiency, the simulation results prove that joining V-BLAST with STTC improves MIMO-OFDM systems performance.
\end{abstract}

Index terms- V-BLAST, STTC, BLAST-STTC, MIMOOFDM

\section{INTRODUCTION}

MIMO (multiple input multiple output) is the use of multiple antennas at both the transmitter and receiver to improve communication performance. The latter has been proved efficient in flat fading channel in implementation of the standard IEEE 802.16 as well as the future communication generation.

Depending on transmission techniques, MIMO is classified into multiplexing and space time coding. Among MIMO multiplexing techniques we have VBLAST, this technique provides the high capacity and spectral efficient, however, this has the drawback of fading. Whereas in MIMO space time coding techniques we have STBC (space time block coding) which presents full diversity gain; nonetheless, it lacks the coding gain. On the other hand, STTC which is obtained by a joint design of error control coding, modulation, transmitting and receiving diversity to combat the effects of fading, the drawback of this, is the design complexity which increases as STTC state and the number of antenna increase.

OFDM (orthogonal frequency division multiplexing)

\footnotetext{
Manuscript received July 1, 2010; accepted July 1, 2010.

Christine is a student in the Department of Electric and Electronic Engineering, Korea University of Technology and Education, Cheonan,330-708,Korea (Email: nchristine8@gmail.com)
}

transforms selective frequency fading channel which is a characteristic of the wireless broadband, into parallel flat fading channel that is less complex to combat [1]. Hence, combining MIMO and OFDM allows the deployment of wireless broadband access.

In MIMO-OFDM system, V-BLAST has been proposed for high capacity and spectral efficient in wireless communication. However, its performance is critical when resisting to fading. To handle this issue, space time coding such as STBC and STTC has been proposed. Despite of providing full diversity gain, STBC is lacking the coding gain, hence we propose STTC. By introducing spatial and temporal correlation into the signals transmitted from different antennas, STTC achieves both diversity gain and coding gain. STTC can simultaneously offer substantial coding gain, spectral efficiency and diversity gain on fading channels [2]. In this paper, STTC is proposed to be joined with VBLAST; however, as mentioned above, STTC design complexity gets high as the number of antennas increases. Therefore, we group the antennas into small group with generally not high number of antennas. In this paper, the group is composed of 2 antennas; what this system differs from traditional V-BLAST is that, it uses multiple antennas in each layer, whereas in traditional V-BLAST system all antennas belong to one group, which means that there is no way to split them into small group. In this paper, we use layer and group interchangeably.

In order to reduce the receiver complexity and to guarantee each group' $\mathrm{s}$ full diversity, the group interference cancellation method is used before decoding with STTC decoder. The same algorithm has been used in [3]. However, it considers single carrier. This joint system obtains both diversity gain and coding gain without sacrificing bandwidth efficiency. Therefore, the integration of this combination has the potential to meet the ever growing demands of future communication systems.

The outline of this paper is as follows, section II presents the system model, section III introduces the detection design, and section IV evaluates and analyzes the system's performance through simulation result. Finally, conclusions on the presented work are drawn in section $\mathrm{V}$. 


\section{SYSTEM MODEL}

The system has $\mathrm{N}$ transmit antennas and $\mathrm{M}$ receive antennas over quasi static frequency selective fading channel. As the figure 1 shows, $\mathrm{B}$ is the input bits block flow of the system; the data to be transmitted are encoded by STTC encoder. As we are comparing MIMO-OFDM system with and without STTC, this system without STTC will be encoded by convolutional encoder.

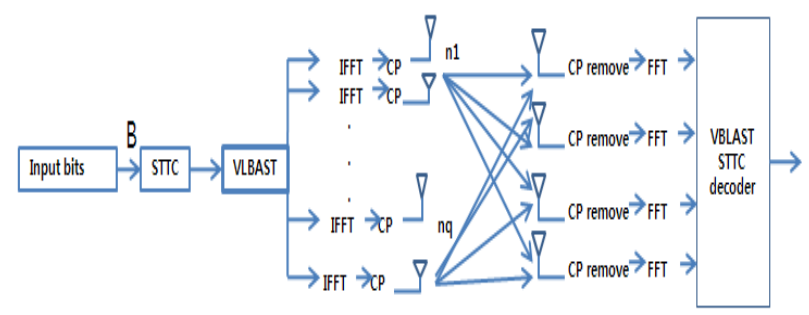

Fig. 1. Block diagram of V-BLAST STTC in MIMO-OFDM.

STTC is the extension of conventional trellis codes to multi-antenna systems [4]. The number of nodes in a trellis diagram corresponds to the number of states in the trellis. Figure 2 shows a simple QPSK (quadrature phase shift keying) constellation, four state trellis code for $M_{T}=2$. In this paper, $M_{T}$ represents the number of transmit antennas of every group, with rate 2 symbols/s/Hz each group.

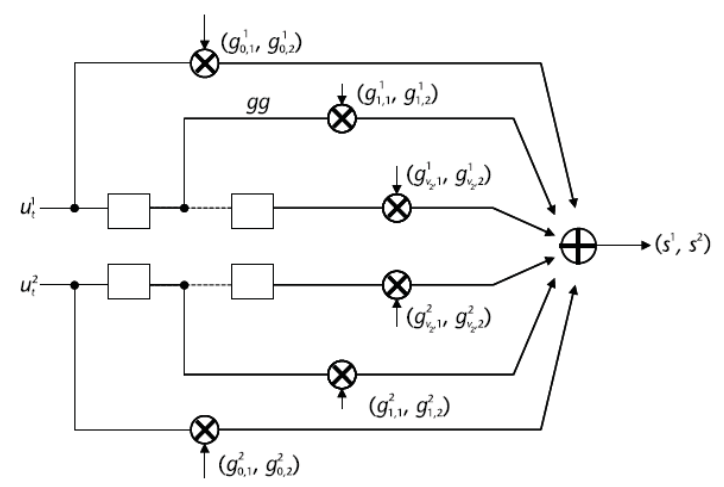

Fig. 2. Space-time trellis code encoder for QPSK.

As it is shown on figure $3, s^{1}$ and $s^{2}$ symbols come in pairs wherein the first digit corresponds to the symbol transmitted from antenna 1 and the second from antenna 2 of each group.

Both STTC and convolutional coding use trellis coding and viterbi algorithm for decoding as well as error control techniques. However, convolutional coding does not include modulation as it is the case for STTC.

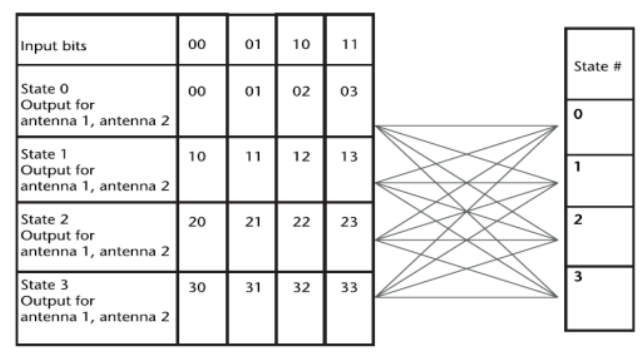

Fig. 3. Example of 2 transmit space-time trellis code with 4 states (QPSK constellation with spectral efficiency of 2 symbols $/ \mathrm{s} / \mathrm{Hz}$ ).

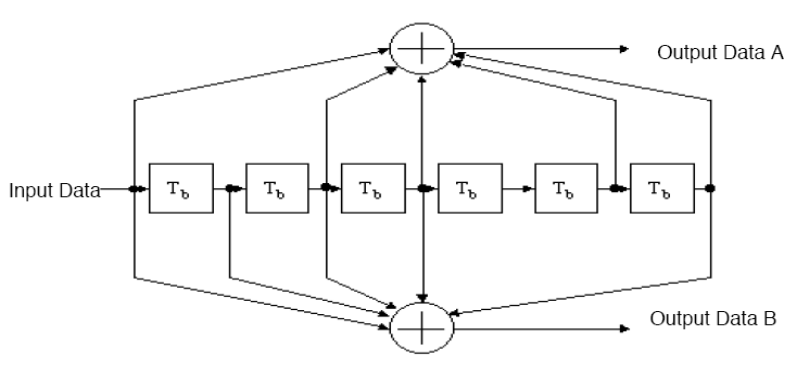

Fig. 4. Convolutional encoder (constraint length $\mathrm{k}=7$ ).

At each instant $t$, block of $q$ binary information symbols denoted by $B_{t}$ is fed into STTC encoder.

$$
B_{t}=\left(s_{t}^{1}, s_{t}^{2}, \ldots, s_{t}^{q}\right) .
$$

STTC encoder maps the block B binary input data of length q into $M_{T}$ modulation symbols and forms signal set of $M=2^{q}$ points.

The coded data are applied to a serial to-parallel(S/P) converter to produce a sequence of $M_{T}$ parallel symbols, arranged as a $M_{T} \mathrm{X} 1$ column vector $\mathrm{S}_{\mathrm{t}}$ where $T$ means the transpose of a matrix.

$$
S_{t}=\left(s_{t}^{1}, s_{t}^{2}, \ldots, s_{t}^{M_{T}}\right)^{T} .
$$

The $M_{T}$ parallel outputs coded by BLAST to be spread into transmit antennas before being simultaneously transmitted from all the $M_{T}$ antennas. Transmit antennas are grouped into groups with each belongs to one $\mathrm{V}$ BLAST layer. Whereby symbol $s_{t}^{i}, 1 \leq i \leq M_{T}$ is transmitted by antenna $n_{i}$ of the group and all transmitted symbols have the same duration of $T$ seconds. The vector of coded modulation symbols from different antennas, as shown in equation (2), is called a space time symbol. The spectral efficient of the system is given by

$$
\eta=\frac{r b}{B}=q b i t s / s / H z
$$


Where $\mathrm{rb}$ is the data rate and $\mathrm{B}$ is the channel bandwidth. The above spectral efficient is equal to the spectral efficiency of a reference uncoded system with one transmit antenna.

In the situation where we have multiple antennas at both transmitter and receiver, we consider a MIMO channel which is a matrix $\mathrm{H}$ given in equation (6). Thereafter, each sequence from every antenna is modulated by IFFT (inverse fast Fourier transform) .This IFFT changes the signal to be transmitted in time domain. After that, cyclic prefix is added; the cyclic prefix acts as a buffer region where delayed information from the previous symbols can get stored.

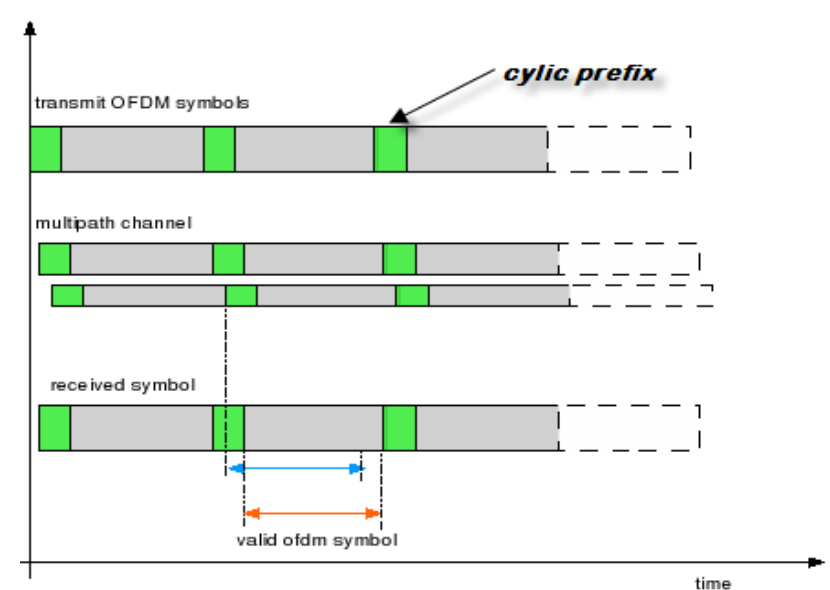

Fig. 5. OFDM symbol with multipath.

Given that transmission of cyclic prefix reduces the data rate, the system designers will want to minimize the cyclic prefix duration. Typically, cyclic prefix duration is determined by the expected duration of the multipath channel in the operating environment. For example, for the indoor wireless multipath channel, the typically expected multipath channel is of around $0.8 \mu \mathrm{sec}$ duration, hence, determining the cyclic prefix chosen per the IEEE 802.11a specification[5], the general length of the cyclic prefix should be at least a quarter(1/4) of the length of the OFDM symbol. In this paper, the length symbol is 64 thus the cyclic prefix length considered is 16 .

Finally, the signal $\mathrm{S}_{\mathrm{n}}[\mathrm{t}, \mathrm{k}], \mathrm{n}=1, \ldots, \mathrm{N}$ are simultaneously transmitted at $\mathrm{k}^{\text {th }}$ subcarrier in the $\mathrm{n}^{\text {th }}$ OFDM block from different antenna groups.

On the receiver, the signal at each antenna is a noisy superposition of $\mathrm{N}$ transmitted signal corrupted by fading channel. The received signal at the $\mathrm{M}^{\text {th }}$ receive antenna is

$r_{m}[t, k]=\sum_{n=1}^{N} H_{m, n}[t, k] S_{n}[t, k]+w_{m}$.

Where $\mathrm{H}_{\mathrm{m}, \mathrm{n}}[\mathrm{t}, \mathrm{k}]$ denotes the channel frequency response at $\mathrm{k}^{\mathrm{th}}$ subcarrier of the ${ }^{\text {th }}$ OFDM block between the $\mathrm{n}^{\text {th }}$ transmit and $\mathrm{m}^{\text {th }}$ receive antennas. $\mathrm{w}_{\mathrm{m}}[\mathrm{t}, \mathrm{k}]$ is the noise matrix with independent complex Gaussian random variable with zero mean and variance $\mathrm{N}_{\mathrm{o}} / 2$ and it is assumed to be independent for different t's, k's.

For the sake of simplicity, we represent the detected signal at the $\mathrm{k}^{\text {th }}$ subcarrier of the $\mathrm{n}^{\text {th }}$ OFDM block by omitting $\mathrm{k}$ and $\mathrm{t}$ index as follows

$$
r=H S+w .
$$

where $S=\left(s_{1}, \cdots, s_{N}\right)^{T}, r=\left(r_{1}, \cdots, r_{M}\right)^{T}$, $w=\left(w_{l}, \cdots, w_{M}\right)^{T}$.

$$
H=\left[\begin{array}{ccc}
H_{1,1} & \ldots & H_{1, N} \\
\cdot & & \cdot \\
\cdot & \cdot & \cdot \\
\cdot & & \cdot \\
H_{M, 1} & \ldots & H_{M, N}
\end{array}\right] .
$$

Where the coefficient $H_{i, j}$, which is assumed to be constant during a frame and varies from one frame to another, is the path gain between $i$ transmit antenna and $j$ receive antenna. The coefficients are assumed to be independent and identically distributed Gaussian.

As it is shown above, each layer is composed of $n_{q}$ transmit antennas, transmitted signal $S$ vector can be written as $S=\left(s_{1}, s_{2}, \cdots, s_{n}\right)^{T}$.

Afterwards, the cyclic prefix is removed from the received signal followed by the FFT demodulation; the demodulated $\mathrm{q}$ flows are fed into V-BLAST decoder where the group interference cancellation is done, then the detected signal is decoded by STTC decoder.

\section{GROUP DETECTION ALGORITHM}

At the receiver, group IS (interference suppression) and IC (interference cancellation) are done to recover the original data, which means that we detect one group by suppressing the interference from others without loss of originality.

For instance, let's take the decoding process of the first group and call it $c_{l}$, the group IS method is as follows: the transmit signal vector $\mathrm{S}$ is decomposed into the desired signal and interfering one.

$$
S=S_{\Omega}\left(c_{1}\right)+S_{\Lambda}\left(c_{1}\right) .
$$

Where $S_{\Omega}\left(c_{1}\right)$ is the desired signal and $S_{\Lambda}\left(c_{1}\right)$ is the interfering signal

$$
S_{\Omega}\left(c_{1}\right)=(\underbrace{S_{1}, S_{2}, \ldots S_{n_{1}}}_{n_{1}} 00 \ldots 0)^{T} .
$$




$$
S_{\Lambda}\left(c_{1}\right)=(00 \ldots 0 \underbrace{S_{n_{1}+1}, S_{n_{1}+2} \ldots S_{N}}_{N-n_{1}})^{T} .
$$

Similarly, the channel frequency response matrix is decomposed into two matrices with one corresponding to the desired layer and the other to the interfering layer.

$$
H=H_{\Omega}\left(c_{1}\right)+H_{\Lambda}\left(c_{1}\right)
$$

Where, $H_{\Omega}\left(c_{1}\right)$ is the desired layer channel and $H_{\Lambda}\left(c_{1}\right)$ is the interfering channel from other layer. Where,

$$
\begin{aligned}
& H_{\Omega}\left(c_{1}\right)=\left\{\begin{array}{cc}
H_{1,1} \ldots H_{1, n_{1}} & 00 \ldots 0 \\
\cdot & \ldots \ldots \ldots \\
\ldots \ldots \ldots \ldots & \ldots \ldots . . \\
\underbrace{H_{M, 1} \ldots H_{M, n_{1}}}_{n_{1}} & 00 \ldots 0 \\
N-n_{1}
\end{array}\right\} .
\end{aligned}
$$

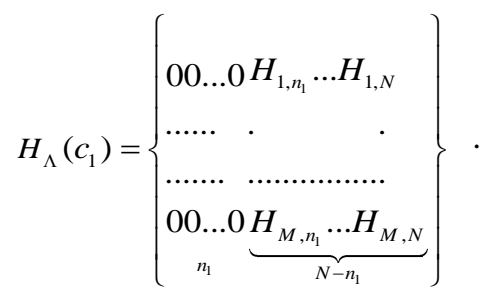

The received signal is as follows

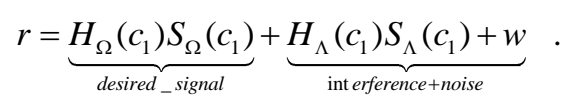

We assume that the CSI is known at the receiver and $M \geq N$. The nulling matrix of $H_{\Lambda}\left(c_{1}\right)$ exists and is denoted by $\left(M-N+n_{1}\right) x M$ matrix $\Lambda\left(c_{1}\right)$, with its rows being the orthonormal basis of the nulling space of $H_{\Lambda}\left(c_{1}\right)$. Therefore $\Lambda\left(c_{1}\right) H_{\Lambda}\left(c_{1}\right)=0$

By multiplying both sides of equation (10), we get

$$
\begin{aligned}
\tilde{r} & =\Lambda\left(c_{1}\right) r \\
& =\Lambda\left(c_{1}\right) H_{\Omega}\left(c_{1}\right) S_{\Omega}\left(c_{1}\right)+\underbrace{\Lambda\left(c_{1}\right) H_{\Lambda}\left(c_{1}\right)}_{=0} S_{\Lambda}\left(c_{1}\right)+\Lambda\left(c_{1}\right) w .
\end{aligned}
$$

If we suppress the interfering layers we get equation (15) with $\tilde{H}_{\Omega}\left(c_{1}\right)=\Lambda\left(c_{1}\right) \tilde{H}_{\Omega}\left(c_{1}\right)$ and $\tilde{w}=\Lambda\left(c_{1}\right) w$.

$$
\tilde{r}=\tilde{H}_{\Omega}\left(c_{1}\right) S_{\Omega}\left(c_{1}\right)+\tilde{w}
$$

As the diversity loss is compensated by detecting the groups with the largest post nulling SNR(signal/noise ratio), we choose the decision group that has the highest post-detection SNR to decode at this level. Thus the detection order is decided by

$$
\beta_{\mathrm{P}}=\frac{\left\|\tilde{H}_{\Omega}\left(c_{P}\right)\right\|_{F}^{2}}{\sigma_{\tilde{w}}^{2}}=\frac{\left\|\Lambda\left(c_{P}\right) H_{\Omega}\left(c_{P}\right)\right\|_{F}^{2}}{\sigma_{\tilde{w}}^{2}} .
$$

with $\mathrm{p}=1,2 \ldots \mathrm{Q}$

where $\|$.$\| is the frobenius norm and \sigma^{2}{ }_{\tilde{w}}$ is the variance of noise after group interference suppression. The first group to be detected is found by

$$
p_{0}=\arg \max \left(\beta_{\mathrm{P}}\right) \text {. }
$$

The detection of the each layer can achieve the spatial diversity order of $\left(M-N+n_{i}\right) x n_{i}$.Then; this layer is decoded by STTC decoder. Furthermore, the contribution of the detected $\mathrm{k}^{\text {th }}$ layer is cancelled from the received signal and the similar processing is applied to the remaining layers to get them detected sequentially.

\section{SIMULATION AND DISCUSSION}

Assume that the channel fading is constant during a

\begin{tabular}{|c|c|}
\hline Parameter & Type \\
\hline $\begin{array}{l}\text { MIMO-OFDM } \\
\text { type }\end{array}$ & $\begin{array}{l}\text { STTC V-BLAST, } \\
\text { V-BLAST }\end{array}$ \\
\hline $\mathrm{TX} \times \mathrm{RX}$ & $2 \times 2,4 \times 4$ \\
\hline CP length & 16 \\
\hline Frame length & 256 bits \\
\hline $\begin{array}{l}\text { Modulation } \\
\text { scheme }\end{array}$ & 4-QAM \\
\hline Channel coding & $\begin{array}{l}\text { STTC4-states in case of STTC V-BLAST } \\
\text { MIMO-OFDM } \\
\text { Convolutional coding } 1 / 2 \text { in case of } \\
\text { V-BLAST MIMO-OFDM }\end{array}$ \\
\hline FFT length & 64 \\
\hline Channel & Frequency selective fading channel \\
\hline $\begin{array}{l}\text { Generate matrix } \\
\text { Tarokh generate } \\
\text { matrix }\end{array}$ & $G=\left[\begin{array}{llll}0 & 0 & 2 & 1 \\
2 & 1 & 2 & 0\end{array}\right]$ \\
\hline Simulation Tool & Matlab 7.8.0 (R2009a) \\
\hline
\end{tabular}
frame and changes independently from one frame to another. Furthermore, transmit and receive antenna are assumed to be uncorrelated. Through simulation, we evaluate the BER (bit error rate) performance for both systems according to SNR. The system parameters are given below:

TABLE I

SIMULATIONS PARAMETERS 
As it is shown on the figure 2 which considers $2 \times 2$ system, MIMO-OFDM with V-BLAST and STTC-4 states performs better than the system that does not use STTC, instead it uses CC (convolutional coding). When considering the system $4 \times 4$ with transmit antennas grouped into two groups of 2 antennas each, as it is also shown on figure 3 ; we observe that the system with the joint scheme of V-BLAST and STTC is outperforming the system that uses V-BLAST and convolutional coding; this is due to diversity, error control and coding gain obtained from STTC.

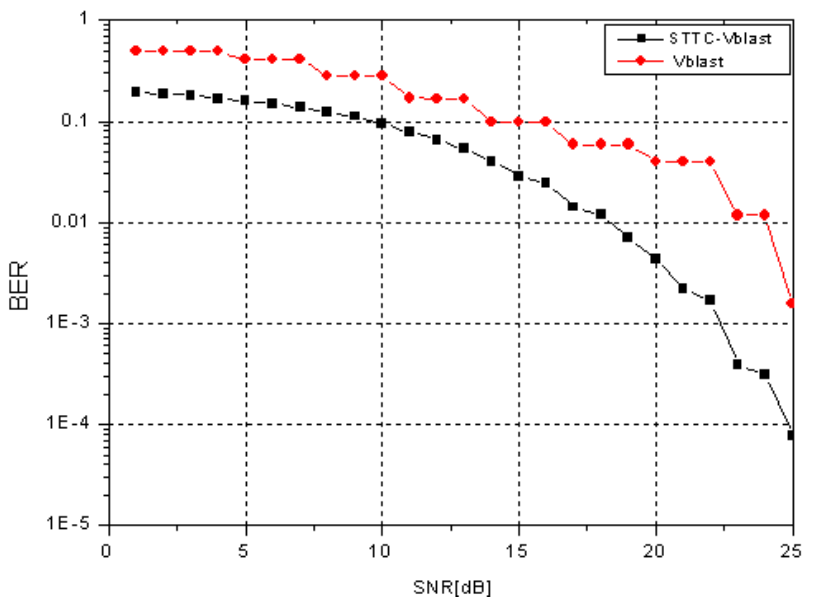

Fig. 6. BER performance of VBLAST STTC over VBLAST considering MIMO-OFDM $2 \times 2$.

By comparing the bit error performance, we find that in the high SNR, the joint system significantly outperforms. For instance, on the figure 2 at SNR that equals to $30 \mathrm{~dB}$, we find that BER for the joint system (2x2) is about $10^{-4}$ whereas we have about $10^{-2}$ for MIMO-OFDM without STTC. In the case of the system $4 \times 4$ we realize that the joint system's BER performance is about $10^{-5}$ at SNR equals to $30 \mathrm{~dB}$ whereas for the system without STTC, it is about 10-2 at same SNR price. With regard to SNR, as this one is increasing, as the difference in BER performance of both systems increases, with the joint system outperforms.

\section{CONCLUSIONS}

In this paper, we have studied the improvement of MIMO-OFDM system efficiency based on combining VB-LAST and STTC; this uses a group of antennas on each V-BLAST layer and the signal interference cancellation technique on the receiver. Through simulation results we confirm that the joint system outperforms the traditional V-BLAST which uses one antenna on each layer. Moreover, the receiver complexity has been reduced based on group interference cancelation to the extent that the complexity of $4 \times 4$ is reduced to $4 \times 2$ at the same time keeping the diversity of $4 \times 4$.

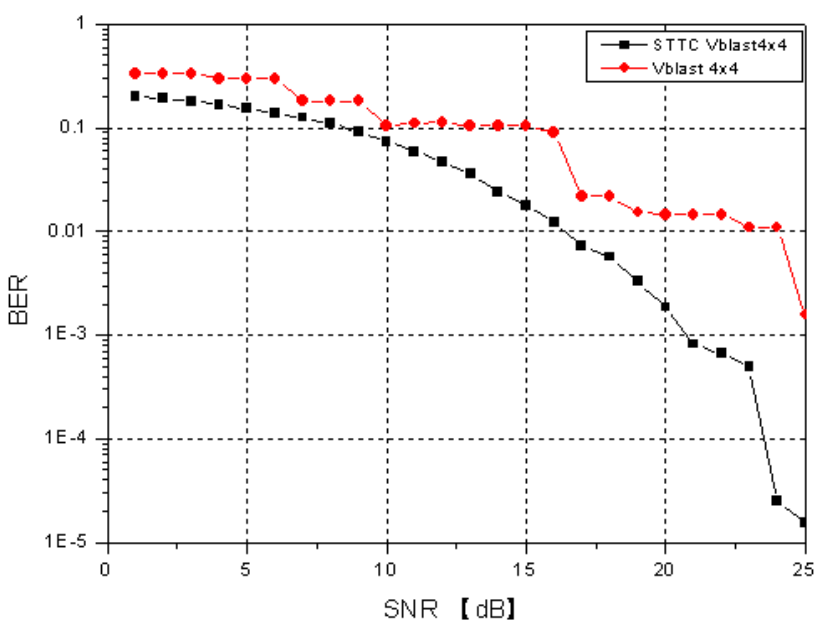

Fig. 7. BER performance of MIMO-OFDM $4 \times 4$.

\section{REFERENCES}

[1] D. Agrawal, V. Tarokh, A. Naguib, and N. Seshardri, "Space-time coded OFDM for high data-rate wireless communication over wideband channels," IEEE Vehicular Technology Conference, 1998.

[2] S. Sandhu, R. Heath, and A. Paulraj, "Space-time block codes versus space-time trellis codes," IEEE ICC, Vol. 4, pp. 1132-1136, 2001.

[3] Y.Dai, Z.Lei and S.Sun, "Ordered array processing for space-time coded systems," IEEE Commun. Lett. vol. 8, pp.526-528, August 2004.

[4] Mohinder jankiraman, "Space-time codes and MIMO systems," Artech House.

[5] Tarokh, Neshadri, and A.R.Calderbank, "Space time codes for high and data rate wireless communication: performance criterion and code construction," IEEE.Trans.Inf, vol. 44, no.2, pp.744-765, 1998.

[6] http://standards.ieee.org/getieee802/download/802.11a-1999.pdf.

[7] G. J. Foschini and M. J. Gans, "On limits of wireless communications in a fading environment when using multiple antennas," Wireless Personal Communication, pp. 311-335, March 1998.

[8] P. W. Wolniansky, G. J. Foschini, G. D. Golden, and R. A.Valenzela, "V-BLAST: An architecture for realizing very high data rates over the rich scattering channel," Proc. ISSE, pp.295300, September 1998.

[9] Xiaoqiang Qiao, Yueming Cai, and Youyun Xu, "Joint iterative decision feedback channel estimation for turbo coded V-BLAST MIMO-OFDM systems," IEEE. Proceedings of ISCIT, 2005.

[10] Xue WEN LLL, Jun WANG, Youxi TANG, and Shaoqian LI, "Lattice reduction-based detection of joint V-BLAST and SFBC in MIMO/OFDM systems," IEEE Proceedings. 2005 International Conference, vol.1, 2005.

[11] J ianhua Zhang, Ruoju Liu, Guangyi Liu, and Ping Zhang, "Multipath delay estimation with interference cancellation in MIMO-OFDM system," Vehicular Technology Conference, IEEE $64^{\text {th }}, 2005$. 


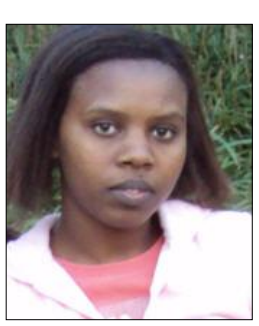

Christine Niyizamwiyitira was born in, Rwanda, on January 06, 1984. She received the B. S. degree in Computer science from National University, Butare, Rwanda, in 2008. She is a master's student in information technology engineering from Korea University of Technology and Education, Chungnam, Korea. Her research interests are in the areas of wireless communications, MIMO-OFDM and mobile communication.

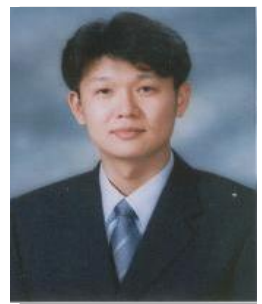

Chul Gyu Kang was born in Daejoen, Korea, on January 31, 1976. He received the B. S. and M.S.E. degrees in information technology engineering from Korea University of Technology and Education, Chungnam, Korea, in 2004 and 2006, respectively. He is a Ph.D. student in information technology engineering from Korea University of Technology and Education, Chungnam, Korea. His research interests are in the areas of wireless communications and wireless sensor networks with particular emphasis on wireless localization.

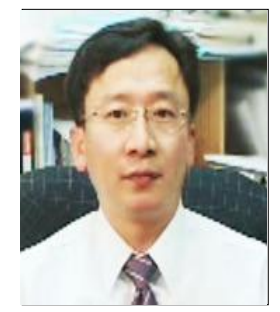

Chang Heon Oh was born in Seoul, Korea, on February 10, 1965. He received the B. S. and M.S.E. degrees in telecommunication and information engineering from Korea Aerospace University, Kyunggi-Do, Korea, in 1988 and 1990, respectively. He received the Ph.D. degree in avionics engineering from Korea Aerospace University, Kyunggi-Do, Korea, in 1996. From February 1990 to August 1993, he was with Hanjin Electronics Co., where he was involved in the research and development of radio communication \& monitoring systems. From October 1993 to February 1999, he was with the CDMA R\&D center of Samsung Electronics Co., where he was involved in the design and development of CDMA cellular systems and CDMA PCS systems for successful commercial CDMA deployment in Korea. Since March 1999, he has been with the School of Information Technology, Korea University of Technology and Education, Chonan, Korea, where he is currently a professor. His research interests are in the areas of wireless communications, mobile communication, and wireless sensor networks with particular emphasis on wireless localization.

Dr. Oh is a member of the IEEE Communication Society, IEEE Vehicular Technology Society, IEEE Signal Processing Society, IEEE Education Society, Korean Institute of Communication Sciences (KICS), Korea Electromagnetic Engineering Society (KEES), Korea Navigation Institute (KONI), Korea Institute of Maritime Information \& Communication Sciences (KIMICS) and Korea Institute for Practical Engineering Education (KIPEE). He is currently serving as an Executive Director for the KONI, KIMICS and KIPEE. He received the Gold Awards from the Samsung Group in 1998. 